University of Nebraska - Lincoln

DigitalCommons@University of Nebraska - Lincoln

Michigan Bovine Tuberculosis Bibliography and

Database

Wildlife Disease and Zoonotics

$11-2004$

\title{
Investigation of the Transmission of Mycobacterium bovis from Deer to Cattle Through Indirect Contact
}

\author{
Mitchell V. Palmer \\ Bacterial Diseases of Livestock Research Unit, National Animal Disease Center, Agricultural Research \\ Service, USDA, Mitchell.Palmer@ars.usda.gov \\ W. Ray Waters \\ Bacterial Diseases of Livestock Research Unit, National Animal Disease Center, Agricultural Research \\ Service, USDA \\ Diana L. Whipple \\ Bacterial Diseases of Livestock Research Unit, National Animal Disease Center, Agricultural Research \\ Service, USDA
}

Follow this and additional works at: https://digitalcommons.unl.edu/michbovinetb

Part of the Veterinary Medicine Commons

Palmer, Mitchell V.; Waters, W. Ray; and Whipple, Diana L., "Investigation of the Transmission of Mycobacterium bovis from Deer to Cattle Through Indirect Contact" (2004). Michigan Bovine Tuberculosis Bibliography and Database. 72.

https://digitalcommons.unl.edu/michbovinetb/72

This Article is brought to you for free and open access by the Wildlife Disease and Zoonotics at DigitalCommons@University of Nebraska - Lincoln. It has been accepted for inclusion in Michigan Bovine Tuberculosis Bibliography and Database by an authorized administrator of DigitalCommons@University of Nebraska Lincoln. 


\title{
Investigation of the transmission of Mycobacterium bovis from deer to cattle through indirect contact
}

\author{
Mitchell V. Palmer, DVM, PhD; W. Ray Waters, DVM, PhD; Diana L. Whipple, MS
}

\begin{abstract}
Objective-To investigate the infection of calves with Mycobacterium bovis through oral exposure and transmission of $M$ bovis from experimentally infected white-tailed deer to uninfected cattle through indirect contact.
\end{abstract}

Animals-24 11-month-old, white-tailed deer and 28 6-month-old, crossbred calves.

Procedure - In the oral exposure experiment, doses of $4.3 \times 10^{6}$ CFUs (high dose) or $5 \times 10^{3}$ CFUs (low dose) of $M$ bovis were each administered orally to 4 calves; as positive controls, 2 calves received $M$ bovis $\left(1.7 \times 10^{5}\right.$ CFUs) via tonsillar instillation. Calves were euthanatized and examined 133 days after exposure. Deer-to-cattle transmission was assessed in 2 phases (involving 9 uninfected calves and 12 deer each); deer were inoculated with $4 \times 10^{5} \mathrm{CFUs}$ (phase I) or $7 \times 10^{5}$ CFUs (phase II) of $M$ Bovis. Calves and deer exchanged pens (phase I; 90 days' duration) or calves received uneaten feed from deer pens (phase II; 140 days' duration) daily. At completion, animals were euthanatized and tissues were collected for bacteriologic culture and histologic examination.

Results - In the low- and high-dose groups, 3 of 4 calves and 1 of 4 calves developed tuberculosis, respectively. In phases I and II, 9 of 9 calves and 4 of 9 calves developed tuberculosis, respectively.

Conclusions and Clinical Relevance-Results indicated that experimentally infected deer can transmit $M$ bovis to cattle through sharing of feed. In areas where tuberculosis is endemic in free-ranging whitetailed deer, management practices to prevent access of wildlife to feed intended for livestock should be implemented. (Am J Vet Res 2004;65:1483-1489)

I 1994, tuberculosis caused by Mycobacterium bovis infection was diagnosed in a free-ranging whitetailed deer (Odocoileus virginianus) in Michigan. ${ }^{1}$ Subsequent surveys conducted by the Michigan Department of Natural Resources and Michigan State University Animal Health Diagnostic Laboratory identified a focus of $M$ bovis infection in free-ranging whitetailed deer in northeast Michigan. ${ }^{2,3}$ This represented

Received January 12, 2004.

Accepted April 1, 2004

From the Bacterial Diseases of Livestock Research Unit, National Animal Disease Center, Agricultural Research Service, USDA, 2300 Dayton Ave, Ames, IA 50010

Names are necessary to report factually on available data; however, the USDA neither guarantees nor warrants the standard of the product, and the use of the name by USDA implies no approval of the product to the exclusion of others that may also be suitable.

The authors thank Rebecca Lyon, Jody Mentele, Lori Dethloff, Kevin Esch, Shelly Zimmerman, Jessica Pollock, Ryan Cook, and Peter Lasley for technical assistance.

Address correspondence to Dr. Palmer. the first known reservoir of $M$ bovis in free-ranging wildlife in the United States and a major obstacle to the eradication of bovine tuberculosis. Several factors are believed to have contributed to the establishment and persistence of $M$ bovis in this wildlife reservoir. It is postulated that $M$ bovis was transmitted from cattle to deer at some time during the early to mid 1900s when a large number of cattle in Michigan were infected with $M$ bovis. ${ }^{4}$ During this same period, the deer population in Michigan was steadily increasing beyond normal habitat carrying capacity to focal concentrations of 19 to 23 deer $/ \mathrm{km}^{2}$. ${ }^{1}$ Transmission and maintenance of $M$ bovis among deer are believed to have been facilitated by the common practice in Michigan of long-term winter feeding of large volumes of sugar beets, carrots, corn, apples, pumpkins, and pelleted feed to deer by private citizens to prevent migration and decrease the death rate during winter, in an attempt to keep deer numbers high for hunting purposes. ${ }^{1}$ Results of DNA analysis of $M$ bovis isolates from Michigan white-tailed deer indicated that most deer were infected with a common strain of $M$ bovis, which suggested a single source of infection. ${ }^{5}$ The presence of $M$ bovis in wildlife is not only detrimental to the health of the wildlife population but also represents a serious threat to domestic livestock. Thirty-two M bovis-infected cattle herds have been identified in Michigan since the identification of tuberculosis in white-tailed deer. Restriction fragment length polymorphism analysis of $M$ bovis isolates from deer and cattle revealed that they are identical, suggesting that cattle became infected through contact with free-ranging white-tailed deer. ${ }^{a}$

Little is known about the ability of deer to transmit $M$ bovis among themselves or to other susceptible hosts. The purpose of the study reported here was to investigate the infection of calves with $M$ bovis through oral exposure and transmission of $M$ bovis from experimentally infected white-tailed deer to uninfected cattle through indirect contact.

\section{Materials and Methods}

Animals-Twenty-eight 6-month-old crossbred calves were obtained from a herd with no known history of tuberculosis in a state designated as free of bovine tuberculosis. Ten calves were included in the phase of the study to evaluate the establishment of infection with $M$ bovis in cattle through oral exposure, whereas 18 calves were included in the deer-to-cattle transmission phases of the study.

Twenty-four 11-month-old white-tailed deer were obtained from a tuberculosis-free research herd at the National Animal Disease Center in Ames, Iowa, for inclusion in phases I or II of the deer-to-cattle transmission studies (12 deer/phase). All animals were observed twice daily by animal care or veterinary staff. A protocol detailing experimental 
procedures and animal care was approved by the Institutional Animal Care and Use Committee prior to the experiment.

Preparation of the inoculum-The mycobacterial inoculum was prepared from a recent white-tailed deer isolate of $M$ bovis (strain 1315); this inoculum was administered to both deer and calves. The inoculum consisted of mid-logphase $M$ bovis grown in Middlebrook $7 \mathrm{H} 9$ liquid medium with $10 \%$ oleic acid-albumin-dextrose complex ${ }^{b}$ plus $0.05 \%$ Tween $80^{\circ}$ for 10 days at $37^{\circ} \mathrm{C}$. To harvest bacilli from the culture medium, cells were pelleted by centrifugation at $750 \times \mathrm{g}$ and washed twice with $1 \mathrm{~mL}$ of PBS solution $(0.01 \mathrm{M}$; pH 7.2). The inoculum was stored in $1-\mathrm{mL}$ aliquots at $-80^{\circ} \mathrm{C}$ until used. Twenty-four hours after freezing, a single 1-mL aliquot was removed, and bacilli were enumerated via plate counting of serial dilutions prepared with Middlebrook 7H11 medium. At the time of inoculation, aliquots of the inoculum were thawed and diluted to the appropriate concentration (determined from results of the aforementioned plate counting). Each day as the inoculum was prepared, plate counts were repeated to confirm the inoculum dose.

Oral inoculation of calves-To evaluate whether cattle could become infected through oral exposure to $M$ bovis and determine the dose required for oral inoculation, 10 calves were randomly assigned to 1 of 3 groups: a group that received a high dose of inoculum orally (high-dose group; $n$ $=4$ ), a group that received a low dose orally (low-dose group; 4 ), or a group that received a single dose of inoculum instilled into the tonsillar crypts (positive control group; 2). On 5 consecutive days, the high-dose group received 8.6 $\times 10^{5} \mathrm{CFUs}$ and the low-dose group received $9.9 \times 10^{2} \mathrm{CFUs}$ of $M$ bovis, resulting in total doses of $4.3 \times 10^{6} \mathrm{CFUs}$ and $5 \times 10^{3} \mathrm{CFUs}$, respectively. Each daily dose of inoculum was prepared immediately before inoculation. The inoculum was suspended in $1 \mathrm{~mL}$ of PBS solution and mixed thoroughly with $10 \mathrm{~g}$ of shelled corn inside a 50-mL polypropylene tube. The corn-inoculum mixture was added to a plastic bag containing $100 \mathrm{~g}$ of pelleted feed. The entire mixture was fed individually to each calf to ensure complete consumption. Calves in the positive control group received a single dose of $1.7 \times 10^{5}$ CFUs of $M$ bovis instilled into the tonsillar crypts, as described ${ }^{6}$ Calves of the same group were housed in pairs in pens. One hundred thirty-three days after inoculation, the calves were euthanatized via IV injection of sodium pentobarbital and specimens of various tissues were collected for bacteriologic culture and histologic examination.

Inoculation of deer-Deer were randomly assigned for inclusion in phase I or II of the study. Deer were experimentally inoculated via intratonsillar inoculation with $4 \times 10^{5}$ CFUs (for inclusion in phase I) or $7 \times 10^{5}$ CFUs (for inclusion in phase II) of $M$ bovis strain 1315, as previously described. ${ }^{7}$ Inoculum was prepared immediately before inoculation as described for use in oral inoculation of the calves. In each phase of the study, 12 deer were used as a source of infection for 9 calves.

Animal housing-In phases I and II of the study, groups of 4 deer or 3 calves were each housed in pens of approximately $16 \mathrm{~m}^{2}$ inside a biosecurity level 3 (BL-3) building. Directional airflow inside the BL-3 building was designed to prevent pen-to-pen transfer of air. Air entering and exiting the pens was passed through high-efficiency particulate air filters. Airflow was controlled to provide 10.4 air changes/h in the animal pens. Animals in each pen had access to a circulating watering device and were fed pelleted feed. Watering devices and feed bunks were separated by a distance of approximately 2.5 to $3.5 \mathrm{~m}$. Protocols and procedures for feeding and cleaning were designed to prevent room-to-room transfer of $M$ bovis.
Indirect contact of deer and calves-Phases I and II of the study were initiated 14 days after experimental inoculation of deer. In phase I, calves were transferred to deer pens and deer were transferred to calf pens on a daily basis; in phase II, uneaten feed was moved from the pens housing inoculated deer to the pens housing calves. In both phases, the same group of 4 inoculated deer switched pens or shared feed with the same group of 3 calves.

To transfer the deer and cattle between pens during phase I, deer were removed to a holding pen while cattle were moved to the soiled pens previously occupied by deer. The pens previously occupied by cattle were thoroughly cleaned by washing the floor and lower walls. Deer were then moved to the cleaned calf pens. During this process, deer and calves had contact only with their pen mates and not other groups of deer or calves. Excess feed had been offered to the deer to ensure that feed remained in the pen when calves entered; approximately $25 \%$ to $50 \%$ of the daily ration remained in the feed bunk at the time of movement. Switching of pens continued for 90 days after which deer were euthanatized via IV injection of sodium pentobarbital for examination; however, calves were euthanatized via IV injection of sodium pentobarbital and examined after an additional period of 90 days (ie, at 180 days) during which no pen transfers occurred.

In phase II, excess feed was offered to experimentally inoculated deer each morning. Approximately 6 to 8 hours later, remaining feed ( $25 \%$ to $50 \%$ of daily ration) was moved from the deer pens to the calf pens. By the next morning, calves had consumed all the feed uneaten by the deer. In this phase of the study, the only contact between deer and cattle was via shared feed and no movement of animals between pens occurred. Sharing of feed continued for 140 days after which deer and calves were euthanatized via IV injection of sodium pentobarbital for examination.

Tuberculin skin testing-Prior to the study, all calves underwent ID tuberculin skin testing by use of the caudal fold test (CFT) or the comparative cervical test (CCT) as described $^{8}$ to detect prior exposure to $M$ bovis. The 10 calves included in the oral inoculation phase of the study were evaluated by use of CCTs at 60 and 120 days after inoculation. Calves from phase I of the deer-to-cattle transmission experiments were evaluated by use of CCTs at 77 and 174 days after the exchange of pens began. Calves from phase II did not undergo skin testing after sharing of feed was initiated. Results were used to categorize calves as negative, suspect, or reactor with regard to exposure to $M$ bovis according to USDA guidelines for skin testing in cattle. ${ }^{8}$

Bacteriologic cultures-In deer involved in phase I of the deer-to-cattle transmission, swab specimens were collected from the oral cavity, nasal cavity, and rectum prior to inoculation, every 30 days thereafter, and at necropsy. In all deer, swab specimens of the tonsillar crypt region were collected prior to inoculation and at necropsy. Swab samples were processed as described. ${ }^{7}$ Additionally, during phase I, samples of pelleted feed, water, and feces from the floor of the deer pens were collected prior to inoculation and every 30 days thereafter. Samples were processed for bacteriologic culture as described. ${ }^{9}$ Bacteriologic culture results were considered positive if $M$ bovis was isolated.

Interferon- $\gamma$ analysis-During the oral inoculation phase and both deer-to-cattle transmission phases of the study, cell-mediated responses to $M$ bovis were monitored via analysis of antigen-specific interferon- $\gamma$ production by use of a commercially available assay ${ }^{\mathrm{d}}$ for the diagnosis of $M$ bovis infection in cattle. Blood was collected from calves in the oral inoculation phase of the study prior to inoculation and on days $36,64,67,99,127$, and 130 after inoculation. Similarly, 
blood was collected from calves in phase I of the deer-to-cattle transmission study prior to the study and on days 28,56 , $77,80,121,140,174$, and 177 after exchange of pens began. Blood from calves in phase II of the deer-to-cattle transmission study was collected prior to the study and on days 45 , 75,103 , and 140 after sharing of feed began. Blood samples were processed within 6 hours of collection, as described. ${ }^{10,11}$ Blood samples were maintained at room temperature (approximately $20^{\circ} \mathrm{C}$ ) prior to analysis. Briefly, blood was collected in $10-\mathrm{mL}$ vacuum tubes containing heparin as anticoagulant. For each calf, at each time point, $1.5 \mathrm{~mL}$ of whole blood was dispensed into separate $2-\mathrm{mL}$ microcentrifuge tubes containing either $0.1 \mathrm{~mL}$ of $M$ avium purified protein derivative (PPDa; $20 \mu \mathrm{g} / \mathrm{mL}$ final concentration), $0.1 \mathrm{~mL}$ of $M$ bovis purified protein derivative (PPDb; $20 \mu \mathrm{g} / \mathrm{mL}$ final concentration), $0.1 \mathrm{~mL}$ of pokeweed mitogen $(20 \mu \mathrm{g} / \mathrm{mL}$ final concentration), or $0.1 \mathrm{~mL}$ of PBS solution. After addition of PPDa, PPDb, pokeweed mitogen, or PBS solution, tubes were capped, mixed by inversion or gentle vortexing, and incubated for 24 hours at $37^{\circ} \mathrm{C}$ in a humidified chamber with $5 \%$ carbon dioxide. After incubation, samples were centrifuged and plasma was collected; plasma samples were stored at $-20^{\circ} \mathrm{C}$ until analyzed. Plasma samples were analyzed for the presence of interferon- $\gamma$ by use of the aforementioned commercially available sandwich enzyme immunoassay, ${ }^{\mathrm{b}}$ according to manufacturer's instructions. Optical density measurements were performed in duplicate at $450 \mathrm{~nm}$ by use of an automated microplate reader. Results were considered positive (ie, indicative of $M$ bovis infection) when the mean optical density value for duplicate samples for PPDb-stimulated plasma was more than 0.05 greater than that of plasma stimulated with PPDa and PBS solution.

Postmortem evaluations-After euthanasia, specimens were collected for bacteriologic culture including the nasal turbinate; tonsil; trachea; lung; liver; spleen; kidney; brain; urine; and mandibular, parotid, medial retropharyngeal, superficial cervical, tracheobronchial, mediastinal, mesenteric, hepatic, iliac, popliteal, and subiliac lymph nodes. Specimens for bacteriologic culture were placed individually in sterile bags and stored at $-80^{\circ} \mathrm{C}$ until processed. Processing of specimens was done as described. ${ }^{12}$ Results were considered positive for infection if $M$ bovis was isolated.

Samples for microscopic examination were obtained from those tissues collected for bacteriologic culture. Specimens were fixed in neutral-buffered $10 \%$ formalin and processed by routine paraffin embedment techniques. Sections were cut ( $5 \mu \mathrm{m}$ thick), stained with H\&E, and examined via light microscopy. Adjacent $5-\mu \mathrm{m}$ sections were cut from specimens with lesions suggestive of tuberculosis (eg, caseonecrotic granulomata) and stained by the ZiehlNeelsen technique for acid-fast bacteria. ${ }^{13}$ Microscopic findings were considered positive for infection when acid-fast bacilli were detected within lesions that were consistent with tuberculosis.

\section{Results}

Tuberculin skin testing-Prior to inclusion in the study, all calves were negative for exposure to $M$ bovis as determined by either CFTs or CCTs. All of the calves inoculated orally with either the high or low doses of $M$ bovis were categorized as negative for exposure to $M$ bovis 60 days after inoculation. At 120 days after inoculation, 1 of 4 calves in the low-dose group was categorized as a reactor and another was categorized as suspect. All calves in the high-dose group were categorized as negative for exposure to $M$ bovis at 120 days after inoculation. Both calves inoculated via tonsillar instillation were categorized as reactors at 60 and 120 days after inoculation. In phase I of the deer-to-cattle transmission investigation, all 9 calves were categorized as reactors at 77 and 174 days after the exchange of deer and cattle between pens began.

Interferon- $\boldsymbol{\gamma}$ analysis-Results of the interferon- $\gamma$ analysis performed prior to inclusion in the study indicated that all calves could be considered negative for exposure to $M$ bovis. By use of this analysis, both calves that received intratonsillar inoculation were categorized as positive for $M$ bovis infection by day 64 after inoculation and were $M$ bovis-positive at all subsequent sampling times. Two of 4 calves in the high-dose group were categorized as positive for $M$ bovis infection 36 days after inoculation; 1 of those 2 calves was also $M$ bovis-positive at 127 and 130 days after inoculation. Two of 4 calves in the low-dose group (ie, the same 2 calves that developed skin test reactions that were categorized as suspect or reactor) were $M$ bovis-positive on days 127 and 130 after inoculation.

In phase I of the deer-to-cattle transmission investigation, 3 of 9 calves yielded positive results via interferon- $\gamma$ analysis 28 days after the exchange of pens with inoculated deer began. Nine of 9 calves were positive for $M$ bovis infection 56 days after the exchange of pens began and were also $M$ bovis-positive on all subsequent sampling times (days 77, 80, 121, 140, 174, and 177), with the exception of 2 calves on day 77 . In phase II of the deer-to-cattle transmission investigation, none of the 9 calves were considered positive for $M$ bovis infection 45 days after receiving feed from pens in which inoculated deer were housed, whereas 7 of 9 calves were $M$ bovis-positive 75 and 103 days after the sharing of feed was initiated. All nine calves in phase II were $M$ bovis-positive by 140 days after the sharing of feed began.

Shedding of $M$ bovis by experimentally infected deer in phase I-Mycobacterium bovis was not isolated from any swab specimen collected from any deer prior to experimental inoculation. Only 1 of 12 deer yielded growth of $M$ bovis on bacteriologic culture of a swab of the oral cavity at a single time point, 90 days after experimental inoculation. Interestingly, $M$ bovis was not isolated from other antemortem swab samples. Feed from 1 pen containing experimentally infected deer yielded growth of $M$ bovis on bacteriologic culture 17 days after experimental inoculation. Mycobacterium bovis was not isolated from other feed samples, water, or feces from the floor of the pen at any time during the study.

Postmortem evaluations-In the oral inoculation group, both calves inoculated via an intratonsillar route developed tuberculosis; the medial retropharyngeal and tracheobronchial lymph nodes were most commonly affected. Ingestion of $M$ bovis resulted in tuberculous lesions or $M$ bovis isolation in 3 of 4 calves in the low-dose group and 1 of 4 calves in the highdose group. The most commonly affected tissues after ingestion of $M$ bovis were tracheobronchial and mediastinal lymph nodes and lungs (Table 1). In contrast to calves inoculated via an intratonsillar route, medial retropharyngeal lymph nodes were not affected in 
Table 1-Number of calves with microscopic lesions suggestive of tuberculosis or bacteriologic isolation of Mycobacterium bovis (or both) in tissues obtained at necropsy 133 days after oral inoculation with low $\left(5 \times 10^{3} \mathrm{CFUs}\right)$ or high $\left(4.3 \times 10^{6} \mathrm{CFUs}\right)$ total doses of $\mathrm{M}$ bovis or intratonsillar inoculation with $1.7 \times 10^{5} \mathrm{CFUs}$ of $M$ bovis.

\begin{tabular}{|c|c|c|c|c|c|c|}
\hline \multirow[b]{2}{*}{$\begin{array}{l}\text { Treatment group } \\
\text { Evaluation results }\end{array}$} & \multicolumn{6}{|c|}{ Tissue } \\
\hline & $\begin{array}{l}\text { Mandibular } \\
\text { lymph node }\end{array}$ & $\begin{array}{c}\text { Tracheobronchial } \\
\text { lymph node }\end{array}$ & \multirow[t]{2}{*}{$\begin{array}{l}\text { Mediastinal } \\
\text { lymph node }\end{array}$} & \multirow[t]{2}{*}{ Lung } & \multirow[t]{2}{*}{$\begin{array}{l}\text { Medial } \\
\text { retropharyngeal } \\
\text { lymph node }\end{array}$} & $\begin{array}{l}\text { Palatine } \\
\text { tonsil }\end{array}$ \\
\hline \multicolumn{4}{|l|}{ Low dose group $(n=4)$} & & & \\
\hline$M$ bovis isolation only* & 0 & 2 & 1 & 1 & 0 & 0 \\
\hline Microscopic lesions only† & 0 & 0 & 1 & 0 & 0 & 0 \\
\hline M bovis isolation and lesions $\ddagger$ & 0 & 1 & 1 & 1 & 0 & 0 \\
\hline Total No. of affected calves & 0 & 3 & 3 & 2 & 0 & 0 \\
\hline \multicolumn{7}{|l|}{ High dose group (4) } \\
\hline$M$ bovis isolation only & 0 & 1 & 1 & 0 & 0 & 0 \\
\hline Microscopic lesions only & 1 & 0 & 0 & 0 & 0 & 0 \\
\hline$M$ bovis isolation and lesions & 0 & 0 & 0 & 0 & 0 & 0 \\
\hline Total No. of affected calves & 1 & 1 & 1 & 0 & 0 & 0 \\
\hline \multicolumn{7}{|l|}{ Intratonsillar group (2) } \\
\hline$M$ bovis isolation only & 0 & 0 & 0 & 0 & 0 & 0 \\
\hline Microscopic lesions only & 0 & 0 & 1 & 0 & 0 & 0 \\
\hline$M$ bovis isolation and lesions & 1 & 1 & 1 & 1 & 2 & 1 \\
\hline Total No. of affected calves & 1 & 1 & 2 & 1 & 2 & 1 \\
\hline \multicolumn{7}{|c|}{$\begin{array}{l}{ }^{*} \text { Mycobacterium bovis isolated by bacteriologic culture and no lesions consistent with tuberculosis iden- } \\
\text { tified postmortem. TLesions consistent with tuberculosis detected microscopically but no isolation of } M \text { bovis } \\
\text { via bacteriologic culture. } ¥ M \text { bovis isolated via bacteriologic culture and lesions consistent with tuberculosis } \\
\text { detected microscopically. }\end{array}$} \\
\hline
\end{tabular}

Table 2-Number of calves with microscopic lesions suggestive of tuberculosis or bacteriologic isolation of $M$ bovis (or both) in tissues obtained at necropsy 90 (phase I) or 140 days (phase II) after initiation of indirect contact* with deer experimentally inoculated via tonsillar instillation with $4 \times 10^{5} \mathrm{CFUs}$ (phase I) or $7 \times 10^{5} \mathrm{CFUs}$ (phase II) of $\mathrm{M}$ bovis.

\begin{tabular}{|c|c|c|c|c|c|c|c|}
\hline \multirow[b]{2}{*}{$\begin{array}{l}\text { Treatment group } \\
\text { Evaluation results }\end{array}$} & \multicolumn{7}{|c|}{ Tissue } \\
\hline & Tonsil & $\begin{array}{c}\text { Medial } \\
\text { retropharyngeal } \\
\text { lymph node }\end{array}$ & $\begin{array}{l}\text { Tracheobronchial } \\
\text { lymph node }\end{array}$ & $\begin{array}{l}\text { Mediastinal } \\
\text { lymph node }\end{array}$ & Lung & $\begin{array}{l}\text { Hepatic } \\
\text { lymph } \\
\text { node }\end{array}$ & $\begin{array}{c}\text { Nasal } \\
\text { turbinate }\end{array}$ \\
\hline \multicolumn{8}{|l|}{ Phase I $(n=9)$} \\
\hline$M$ bovis isolation only & 1 & 0 & 2 & 0 & 0 & 1 & 1 \\
\hline Microscopic lesions only† & 0 & 0 & 2 & 1 & 4 & 0 & 0 \\
\hline$M$ bovis isolation and lesions & 0 & 1 & 5 & 7 & 3 & 0 & 0 \\
\hline Total & 1 & 1 & 9 & 8 & 7 & 1 & 1 \\
\hline \multicolumn{8}{|l|}{ Phase II (9) } \\
\hline$M$ bovis isolation only & 0 & 0 & 1 & 1 & 0 & 0 & 0 \\
\hline Microscopic lesions only & 0 & 0 & 3 & 2 & 0 & 0 & 0 \\
\hline$M$ bovis isolation and lesions & 0 & 1 & 0 & 1 & 1 & 0 & 0 \\
\hline Total & 0 & 1 & 4 & 4 & 1 & 0 & 0 \\
\hline
\end{tabular}

calves that ingested $M$ bovis at either the low or high doses. Tuberculous lesions were not seen in nor was $M$ bovis isolated from the parotid, superficial cervical, mesenteric, hepatic, iliac, popliteal, or subiliac lymph nodes; brain; nasal turbinate; trachea; liver; kidney; spleen; or urine from any of the calves inoculated by the intratonsillar or oral routes.

In the deer-to-cattle transmission phases of the study, all experimentally inoculated deer developed disseminated tuberculosis; the tonsils, lungs, and medial retropharyngeal, tracheobronchial, mediastinal, hepatic, and mesenteric lymph nodes were most commonly affected. Affected tissues included those from which $M$ bovis was isolated or that contained microscopic lesions consistent with tuberculosis. Mycobacterium bovis was isolated from 2 to 8 different tissues from each of the experimentally inoculated deer. In most instances, tissues that yielded growth of $M$ bovis via bacteriologic culture contained tuberculous lesions. Other sites affected in experimentally inoculated deer included the trachea; liver; spleen; kidney; and mandibular, parotid, superficial cervical, iliac, subiliac, and popliteal lymph nodes. Tuberculous lesions were not seen nor was $M$ bovis isolated from nasal turbinate or brain samples of any experimentally inoculated deer. Similarly, M bovis was not detected in the urine of experimentally inoculated deer at the time of necropsy. Swab specimens collected at necropsy of the oral cavity and tonsillar crypt obtained from deer used in phase I of the study commonly yielded growth of $M$ bovis via bacteriologic culture (5/12 deer for each swab specimen). Less commonly, $M$ bovis was isolated from swabs 
of the tracheal lumen (3/12 deer) and rectum (1/12 deer). Swabs of the nasal cavity did not yield $M$ bovis from any of the deer in phase I of the study.

Regardless of the form of indirect contact used in deer-to-cattle transmission investigation, the most common sites at which tuberculous lesions were detected or from which $M$ bovis was isolated in calves were the tracheobronchial and mediastinal lymph nodes (Table 2). Tracheobronchial lymph nodes were affected in 9 of 9 calves and 4 of 9 calves in phases I and II, respectively; mediastinal lymph nodes were affected in 8 of 9 calves and 4 of 9 calves in phases I and II, respectively. Lung tissue was commonly affected in calves involved in phase I (7/9), but less so in calves involved in phase II (1/9). Lesions were not detected nor were $M$ bovis organisms isolated via bacteriologic culture of samples of the mandibular, parotid, superficial cervical, mesenteric, iliac, popliteal, or subiliac lymph nodes; trachea; liver; spleen; kidney; or brain from calves in either phase of the deer-to-cattle transmission investigation. Mycobacterium bovis was not isolated from urine samples from any calf in either phase of the study.

\section{Discussion}

Results of our study have indicated that ingestion of as few as $5 \times 10^{3}$ CFUs of $M$ bovis can result in established infection and tuberculous lesions in calves, demonstrating that the oral infectious dose for calves is far lower than that previously estimated. Findings of another study ${ }^{14}$ indicated that at least $1 \times 10^{7}$ CFUs of $M$ bovis are required to establish infection in cattle via the oral route of exposure. Similarly, $1.3 \times 10^{6} \mathrm{CFUs}$ of $M$ bovis administered orally to sheep resulted in development of tuberculosis in some but not all of the animals that were inoculated. ${ }^{15}$ It is possible that $M$ bovis strain 1315 (that was originally isolated from deer and used in our study) is more virulent in cattle than other strains used in earlier studies. However, comparison of the virulence of strain 1315 with that of an $M$ bovis strain originally isolated from dairy cattle did not reveal differences in lesion distribution or severity after aerosol inoculation of cattle. ${ }^{16}$

Our data have indicated that $\mathrm{M}$ bovis can be transmitted from experimentally infected white-tailed deer to cattle through indirect contact. Although all 9 of 9 calves became infected in phase I of the study that involved transferring calves and experimentally inoculated deer between pens, the provision of feed alone from pens of inoculated deer to calves in phase II was sufficient to transmit $M$ bovis to 4 of 9 calves; therefore, contact that is limited to the sharing of feed is sufficient to transmit $M$ bovis from experimentally inoculated deer to cattle.

Results of another study ${ }^{15}$ have suggested that lesions in mesenteric lymph nodes are common in cattle infected with $M$ bovis via oral exposure. In the study of this report, mesenteric lymph nodes were not affected in cattle that were inoculated orally with $M$ bovis or in cattle that had indirect contact with $M$ bovis-inoculated white-tailed deer and were presumably infected via the oral route. Rather, lesions in all calves in our study were predominantly within the tracheobronchial and mediastinal lymph nodes and the lungs. The reason for this lesion distribution is unclear. A similar pattern of lesion distribution was observed in white-tailed deer offered feed that had been exposed to other deer that had been experimentally inoculated with M bovis. ${ }^{17}$ Moreover, localization of lesions primarily in the lungs and associated lymph nodes has been detected in cattle and white-tailed deer after aerosol exposure to $M$ bovis. ${ }^{16,18}$ It is doubtful that aerosol transmission could have played a major role in the study reported here. In the BL-3 building used, air pressure and airflow velocity and direction were monitored continuously to prevent room-to-room transmission of aerosols. However, it is possible that pelleted feed used in our study contained fine feed particles that could have been inhaled during feeding, resulting predominantly in the development of lesions in lungs and associated lymph nodes. The effect of different feed types on the efficiency of indirect transmission of $M$ bovis from deer to cattle through a shared source of feed requires investigation.

The calf-to-calf transmission of $M$ bovis has been investigated in experimentally inoculated or naturally infected calves, and those studies, ${ }^{19-21}$ revealed a pattern of gross lesions in calves similar to that observed in our study. However, in those studies, the number of in-contact calves to develop tuberculosis ranged from $25 \%$ to $40 \%$, depending on the study, in contrast to the $44 \%$ to $100 \%$ transmission of $M$ bovis detected in the study of this report. Differences in findings between our study and the previous studies may be attributable to species differences in shedding of $M$ bovis (ie, deer vs cattle) or differences in source of inoculum or total dose of inoculum, animal density, or environmental conditions. Transmission of $M$ bovis in our study may have been artificially enhanced by the dose of inoculum administered to the experimentally inoculated deer and the confinement of the BL-3 animal housing. The open environment of more natural surroundings would likely decrease the efficiency of disease transmission. Lesions that developed in experimentally inoculated deer in our study were similar to those detected in naturally infected white-tailed deer., $1,2,9$ However, the dose of $M$ bovis that causes infection in free-ranging white-tailed deer or the magnitude of bacterial shedding that occurs from naturally infected white-tailed deer is unknown and likely highly variable; therefore, comparison of conditions of $M$ bovis infection among free-ranging deer with those determined in the study of this report is problematic.

Intermittent shedding of $M$ bovis in saliva, nasal secretions, and, less commonly, urine and feces, as well as the contamination of feed, has been detected in experimentally infected white-tailed deer.22 Low-level or intermittent shedding by infected animals combined with relatively small sample size and infrequent (ie, monthly) collection of samples may explain the low recovery of $M$ bovis from oral and nasal swabs and feed samples in our study. Improved and more sensitive methods of detection may be required to accurately assess environmental contamination by $M$ bovis-infected animals. Mycobacterium bovis has been isolated from tracheal swab specimens and the palatine (oropharyn- 
geal) tonsils obtained from naturally infected whitetailed deer. ${ }^{9}$ Similarly, the organism has been detected in nasal and tracheal swab specimens and the oropharyngeal tonsils obtained from naturally infected red deer (Cervus elaphus) and samples of nasal mucus from naturally infected cattle. ${ }^{23-26}$ Although the number of $M$ bovis bacilli shed by tuberculous white-tailed deer has not been determined, our data have suggested as little as $5 \times 10^{3} \mathrm{CFUs}$ or less may have been shed and transmitted from experimentally infected deer to cattle through the feed. Furthermore, although indirect contact lasted 90 and 140 days in phases I and II of the deer-to-cattle transmission investigation, respectively, monitoring of cell-mediated responses to $M$ bovis revealed that exposure had occurred in at least 3 of 9 calves involved in phase I by day 28 of indirect contact and 7 of 9 calves involved in phase II by day 75 of indirect contact.

Although all 9 of 9 calves involved in phase II of the deer-to-cattle transmission investigation developed cellmediated immune responses consistent with exposure to $M$ bovis, only 4 of 9 calves had lesions consistent with tuberculosis or had tissues from which $M$ bovis was isolated via bacteriologic culture. This discrepancy may be attributable to the low sensitivity of bacteriologic culture of tissues in which few $M$ bovis bacilli are present, bacterial colonization in tissues that were not cultured, resolution of infection by the calves following infection, or false-positive results associated with the interferon- $\gamma$ assay. Although these represent interesting and important issues to resolve through future research, this discrepancy does not invalidate our finding of indirect transmission of $M$ bovis from tuberculous white-tailed deer to cattle through sharing of feed.

In Switzerland, naturally infected roe deer (Capreolus capreolus) are suspected to have transmitted $M$ bovis to domestic cattle through contaminated feed. ${ }^{27}$ Cattle have also become infected via contact with feces or urine from $M$ bovis-infected badgers. ${ }^{28}$ In northern Michigan, where $M$ bovis infection is endemic in freeranging white-tailed deer, feeding of deer during the winter has been incriminated as a means of maintaining infection in this population. ${ }^{1}$ Large numbers of deer around feeding sites provide opportunity for close contact and transmission of $M$ bovis; the feeding sites provide common areas where infected deer may contaminate feed that is consumed by deer or other animals. Likewise, infected deer entering livestock feeding areas may contaminate feed intended for consumption by cattle. Under appropriate conditions, $M$ bovis may persist in the environment for weeks to months. ${ }^{29-31, e}$ Therefore, livestock producers in areas where $M$ bovis is endemic in white-tailed deer should implement management practices to prevent access of wildlife to livestock feeds. Allowing potentially infected deer to share feed intended for livestock will enhance indirect transmission of $M$ bovis to cattle, thereby making disease control more difficult and eradication unlikely.

${ }^{a}$ Whipple DL, Jarnagin JL, Payeur JB. DNA fingerprinting of Mycobacterium bovis isolates from animals in northeast Michigan (abstr), in Proceedings. 9th Int Symp World Assoc Vet Lab Diagn 1999;83.

bleic acid-albumin-dextrose complex (OADC), Difco, Detroit, Mich.
'Sigma Chemical Co, St Louis, Mo.

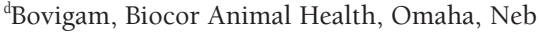

'Whipple DL, Palmer MV. Survival of Mycobacterium bovis on feeds used for baiting white-tailed deer (Odocoileus virginianus) in Michigan (abstr), in Proceedings. 49th Annu Wildl Dis Assoc 2000;21.

\section{References}

1. Schmitt SM, Fitzgerald SD, Cooley TM, et al. Bovine tuberculosis in free-ranging white-tailed deer from Michigan. L Wildl Dis 1997;33:749-758.

2. O'Brien DJ, Fitzgerald SD, Lyon TJ, et al. Tuberculous lesions in free-ranging white-tailed deer in Michigan. L Wildl Dis 2001; 37:608-613.

3. O'Brien DJ, Schmitt SM, Fierke JS, et al. Epidemiology of Mycobacterium bovis in free-ranging white-tailed deer, Michigan, USA, 1995-2000. Prev Vet Med 2002;54:47-63.

4. Frye GH. Bovine tuberculosis eradication: the program in the United States. In: Thoen CO, Steele JH, eds. Mycobacterium bovis infection in animals and humans. Ames, Iowa: Iowa State University Press, 1995;119-129.

5. Whipple DL, Meyer RM, Berry DF, et al. Molecular epidemiology of tuberculosis in wild white-tailed deer in Michigan and elephants, in Proceedings. 101st US Anim Health Assoc 1997;543-546.

6. Palmer MV, Whipple DL, Rhyan JC, et al. Granuloma development in cattle after intratonsilar inoculation with Mycobacterium bovis. Am I Vet Res 1999;60:310-315.

7. Palmer MV, Whipple DL, Olsen SC. Development of a model of natural infection with Mycobacterium bovis in white-tailed deer. I Wildl Dis 1999;35:450-457.

8. USDA. Bovine tuberculosis eradication uniform methods and rules. Washington, DC:US Government Printing Office: 1997.

9. Palmer MV, Whipple DL, Payeur JB, et al. Naturally occurring tuberculosis in white-tailed deer. I Am Vet Med Assoc 2000; 216:1921-1924.

10. Rothel JS, Jones SL, Corner LA, et al. A sandwich enzyme immunoassay for bovine interferon- $\gamma$ and its use for the detection of tuberculosis in cattle. Aust Vet I 1990;67:134-137.

11. Wood PR, Corner LA, Rothel JS, et al. Field comparison of the interferon-gamma assay and the intradermal tuberculin test for the diagnosis of bovine tuberculosis. Aust Vet I 1991; 68:286-290.

12. Payeur JB, Jarnagin JL, Marquardt JG. Laboratory methods in veterinary mycobacteriology for the isolation and identification of mycobacteria. Ames, Iowa; USDA, Animal and Plant Health Inspection Service, National Veterinary Services Laboratories, 1992;5-13.

13. Sheehan DC, Hrapchak BB. Microorganisms. In: Theory and practice of histotechnology. 2nd ed. St Louis: Mosby Publishing Co, 1980; 233-251.

14. Sigurdson J. Studies on the risk of infection with bovine tuberculosis to the rural population. Acta tuberculosea scandinavica supplementum XV. Copenhagen: Munskgaard, 1945;26-40.

15. Francis J. Bovine tuberculosis, including a contrast with human tuberculosis. London: Staples Press Ltd, 1947;86-87.

16. Palmer MV, Water WR, Whipple DL. Aerosol delivery of virulent Mycobacterium bovis to cattle. Tuberculosis 2002;82:275-282.

17. Palmer MV, Waters WR, Whipple DL. Shared feed as a means of deer-to-deer transmission of Mycobacterium bovis. I Wildl Dis 2004:40:87-91.

18. Palmer MV, Waters WR, Whipple DL. Aerosol exposure of white-tailed deer (Odocoileus virginianus) to Mycobacterium bovis. I Wildl Dis 2003;39:817-823.

19. Cassidy JP, Bryson DG, Pollock J, et al. Lesions in cattle exposed to Mycobacterium bovis-inoculated calves. L Comp Pathol 1999; 121:321-327.

20. Neill SD, Hanna J, O'Brien JJ, et al. Transmission of tuberculosis from experimentally infected calves to in-contact calves. Vet Rec 1989;124:269-271

21. Costello E, Doherty ML, Monaghane ML, et al. A study of cattle-to-cattle transmission of Mycobacterium bovis. Vet I 1998; 155:245-250.

22. Palmer MV, Whipple DL, Waters WR. Experimental deerto-deer transmission of Mycobacterium bovis. Am I Vet Res 2001;62:692-696. 
23. Lugton IW, Wilson PR, Morris RS, et al. Epidemiology and pathogenesis of Mycobacterium bovis infection of red deer (Cervus elaphus) in New Zealand. N Z Vet I 1998;46:147-156.

24. de Kantor IN, Bioch D, Roswurm JD. Mycobacteria isolated from nasal secretions of tuberculin test reactor cattle. Am I Vet Res 1978; 39:1233-1234.

25. Neill SD, O'Brien JJ, McCracken, RM. Mycobacterium bovis in the anterior respiratory tracts in the heads of tuberculin-reacting cattle. Vet Rec 1988;122:184-186.

26. Neill SD, Hanna J, Mackie DP, et al. Isolation of Mycobacterium bovis from the respiratory tracts of skin-negative cattle. Vet Rec 1992;131:45-47.

27. Bischofberger VA, Nabholz A. Tuberkulöses wild als ursache von neuinfektionen in rindviehbeständen. Schweiz Arch Tierheilkd 1964;106:759-777.

28. Little TWA, Naylor PF, Wilesmith JW. Laboratory study of Mycobacterium bovis infection in badgers and calves. Vet Rec 1982; 111:550-557.

29. Duffield BJ, Young DA. Survival of Mycobacterium bovis in defined environmental conditions. Vet Microbiol 1985;10:193-197.

30. Jackson R, de Lisle GW, Morris RS. A study of the environmental survival of Mycobacterium bovis on a farm in New Zealand. $\underline{N}$ $Z$ Vet I 1995; 43:346-352.

31. Tanner M, Michel AL. Investigation of the viability of $M$ bovis under different environmental conditions in the Kruger National Park. Onderstepoort I Vet Res 1999;66:185-190. 